\title{
A reduction formula for a $q$-beta integral
}

Zeinab Sayed Mansour ${ }^{1,2}$ and Maryam Ahmed Al-Towailb ${ }^{3 *}$

"Correspondence:

mtowaileb@ksu.edu.sa

${ }^{3}$ Department of Natural and Engineering Science, Faculty of Applied Studies and Community Service, King Saud University, Riyadh, KSA

Full list of author information is available at the end of the article

\section{Abstract}

In this paper, we give a reduction formula for a specific $q$-integral. Our formula is expressed as a three term recurrence relations for basic hypergeometric ${ }_{3} \phi_{2}$ series. This is a q-analog of work by Watson and by Bailey of 1953.

Keywords: q-beta integral; basic hypergeometric series; contiguous relations

\section{Introduction and preliminaries}

In [1], Watson constructed a reduction formula for the integral

$$
I_{n}=\int_{0}^{1} x^{\alpha}(1-x)^{\beta} \frac{d^{n}\left\{x^{\gamma}(1-x)^{\delta}\right\}}{d x^{n}} d x .
$$

In fact, he proved that

$$
I_{n}=\frac{\Gamma(\alpha+\gamma-n+1) \Gamma(\beta+\delta-n+1)}{\Gamma(2 \sigma-n+2)} H_{n},
$$

where

$$
H_{n}=\sum_{r=0}^{n}(-1)^{n-r}\left(\begin{array}{l}
n \\
r
\end{array}\right)(-\gamma)_{n-r}(\beta+\delta-n+1)_{n-r}(-\delta)_{r}(\alpha+\gamma-n+1)_{r},
$$

and the notation $(x)_{r}$ denotes the product

$$
(x)_{0} \equiv 1 \quad \text { and } \quad(x)_{r} \equiv x(x+1) \cdots(x+r-1), \quad r \geq 1
$$

Furthermore, Watson proved that $H_{n}$ satisfies the following three term recurrence relation:

$$
(2 \sigma-n)(\sigma-n) H_{n+2}-Q_{n} H_{n+1}+S_{n} H_{n}=0,
$$

where

$$
\begin{aligned}
& S_{n}=(n+1)(\sigma-n-1)(\alpha+\beta-n)(\gamma+\delta-n)(\beta+\delta-n), \\
& Q_{n}=(2 \sigma-2 n-1)\left\{(\beta \gamma-\alpha \delta)(\sigma+1)+\theta_{3}(n+1)(2 \sigma-n)\right\},
\end{aligned}
$$


and $2 \sigma=\alpha+\beta+\gamma+\delta, 2 \theta_{3}=\alpha-\beta-\gamma+\delta$. Also, he remarked $H_{n}$ can be expressed in terms of a hypergeometric series of the type ${ }_{3} F_{2}$ with last element unity which implies that (1.1) gives a three term contiguous relation for terminating ${ }_{3} F_{2}$ series.

The proof introduced by Watson depends on constructing a second order linear differential equation satisfied by the integrand of $I_{n}$. On the other hand, in [2], Bailey derived relations between contiguous hypergeometric functions of the type ${ }_{3} F_{2}(1)$, and by using these relations, he obtained another proof of Watson's reduction formula.

In this paper, we introduce a $q$-analog of the integral $I_{n}$ by

$$
I_{n, q}=\int_{0}^{1} x^{\alpha}(q x ; q)_{\beta} D_{q^{-1}}^{n}\left[x^{\gamma}\left(q^{\beta+1} x ; q\right)_{\delta}\right] d_{q} x, \quad n=0,1,2, \ldots
$$

where $\alpha, \beta, \gamma$, and $\delta$ are complex numbers, and $q$ is a positive number less than one. Our aim to obtain a reduction formula for $I_{n, q}$.

It turns out to us that Watson technique for introducing (1.1) is too hard to applied to our work. Therefore, we follow Bailey's approach for deriving the reduction formula.

We recall the following definitions (see, e.g., [3-5]):

The $q$-shifted fractional is defined by

$$
(a ; q)_{\infty}=\prod_{j=0}^{\infty}\left(1-a q^{j}\right), \quad \text { and } \quad(a ; q)_{n}:=\frac{(a ; q)_{\infty}}{\left(a q^{n} ; q\right)_{\infty}} \quad \text { for } n \in \mathbb{Z}, a \in \mathbb{C}
$$

The $q$-derivative $D_{q} f$ of an arbitrary function $f$ is given by

$$
\left(D_{q} f\right)(x):=\frac{f(x)-f(q x)}{(1-q) x}, \quad x \neq 0
$$

We follow Gasper and Rahman [6] for the definitions of Jackson $q$-integrals, and the $q$-gamma and $q$-beta functions (see also [7-9]).

The $q$-integration by parts rule (see [3]) is

$$
\int_{0}^{a} f(q t) D_{q} g(t) d_{q} t=f(a) g(a)-\lim _{n \rightarrow \infty} f\left(q^{n}\right) g\left(q^{n}\right)-\int_{0}^{a} D_{q} f(t) g(t) d_{q} t .
$$

Let $a_{1}, \ldots, a_{r}, b_{1}, \ldots, b_{s}$ be complex numbers, the $q$-hypergeometric series ${ }_{r} \phi_{s}$ defined by

$$
{ }_{r} \phi_{s}\left(a_{1}, \ldots, a_{r}, b_{1}, \ldots, b_{s} ; q, z\right)=\sum_{n=0}^{\infty} \frac{\left(a_{1}, \ldots, a_{r} ; q\right)_{n}}{\left(q, b_{1}, \ldots, b_{s} ; q\right)_{n}} z^{n}\left(-q^{(n-1) / 2}\right)^{n(s-r+1)}
$$

The series representation of the function ${ }_{r} \phi_{s}$ converges absolutely for all $z \in \mathbb{C}$ if $r \leq s$, and converges only for $|z|<1$ if $r=s+1$ (for more details and results see [10-14] and [15]).

Observe that

$$
\begin{aligned}
I_{0, q} & =\int_{0}^{1} x^{\alpha+\gamma}(q x ; q)_{\beta+\delta} d_{q} x=B_{q}(\alpha+\gamma+1, \beta+\delta+1) \\
& =\frac{\Gamma_{q}(\alpha+\gamma+1) \Gamma_{q}(\beta+\delta+1)}{\Gamma_{q}(\alpha+\gamma+\beta+\delta+2)}
\end{aligned}
$$


and, by using the $q$-integration by parts, one can verify that

$$
\begin{aligned}
I_{1, q} & =\int_{0}^{1} x^{\alpha}(q x ; q)_{\beta} D_{q^{-1}}\left[x^{\gamma}\left(q^{\beta+1} x ; q\right)_{\delta}\right] d_{q} x \\
& =q^{1-\gamma} \frac{\Gamma_{q}(\alpha+\gamma) \Gamma_{q}(\beta+\delta)}{\Gamma_{q}(\alpha+\gamma+\beta+\delta+1)}\left[[\gamma][\beta+\delta]-q^{\beta}[\delta][\alpha+\gamma]\right],
\end{aligned}
$$

where the notation $[z]$ is defined by

$$
[z]:=\frac{1-q^{z}}{1-q}
$$

Note that the above values of the integrals $I_{0, q}$ and $I_{1, q}$ coincide with $I_{0}$ and $I_{1}$, respectively, which are given by Watson in the limit $q \rightarrow 1$.

This paper is organized as follows. In Section 2, we derive three term contiguous relations for the basic hypergeometric function ${ }_{3} \phi_{2}(a, b, c ; d, e ; q, q)$. In Section 3, we show that $I_{n, q}$ can be represented as ${ }_{3} \phi_{2}(q)$ and a direct substation in the derived contiguous relation yields the result of this paper.

\section{Contiguous relations of ${ }_{3} \phi_{2}$}

Throughout this section, we simply used $a$ to denotes the value $q^{-n}$ where $n$ is an arbitrary nonnegative integer. We denote by $\phi$ the function

$$
{ }_{3} \phi_{2}(a, b, c ; d, e ; q, q) \text {, }
$$

and by $\phi\left(a^{+}\right), \phi\left(a^{-}\right)$the same function when $a$ is changed to $a q, a / q$, respectively. We use a similar notation when the other parameters are so changed. Also, let $\phi_{+}, \phi_{-}$be the functions defined by

$$
\phi_{+}={ }_{3} \phi_{2}(a q, b q, c q ; d q, e q ; q, q) \quad \text { and } \quad \phi_{-}={ }_{3} \phi_{2}(a / q, b / q, c / q ; d / q, e / q ; q, q) .
$$

By the definition of ${ }_{3} \phi_{2}$, one can verify the following:

$$
\begin{aligned}
& \phi\left(a^{+}\right)-\phi=q a \frac{(1-b)(1-c)}{(1-d)(1-e)} \phi_{+}, \\
& \phi\left(a^{-}\right)-\phi=-a \frac{(1-b)(1-c)}{(1-d)(1-e)} \phi_{+}\left(a^{-}\right), \\
& \phi\left(d^{+}\right)-\phi=-q d \frac{(1-a)(1-b)(1-c)}{(1-d)(1-q d)(1-e)} \phi_{+}\left(d^{+}\right), \\
& \phi\left(d^{-}\right)-\phi=d \frac{(1-a)(1-b)(1-c)}{(1-d / q)(1-d)(1-e)} \phi_{+} .
\end{aligned}
$$

These equations, and the symmetries of the ${ }_{3} \phi_{2}$, give us

$$
\begin{aligned}
& c(1-a)\left\{\phi\left(a^{+}\right)-\phi\right\}=a(1-c)\left\{\phi\left(c^{+}\right)-\phi\right\}, \\
& a(1-b)\left\{\phi\left(b^{+}\right)-\phi\right\}=b(1-a)\left\{\phi\left(a^{+}\right)-\phi\right\}, \\
& q a(1-d / q)\left\{\phi\left(d^{-}\right)-\phi\right\}=d(1-a)\left\{\phi\left(a^{+}\right)-\phi\right\},
\end{aligned}
$$




$$
d(1-e / q)\left\{\phi\left(e^{-}\right)-\phi\right\}=e(1-d / q)\left\{\phi\left(d^{-}\right)-\phi\right\} .
$$

Now, applying the transformation (see [6])

$$
{ }_{3} \phi_{2}(a, b, c ; d, e ; q, q)=\frac{(d e / b c ; q)_{n}}{(e ; q)_{n}}(b c / d)^{n}{ }_{3} \phi_{2}(a, d / b, d / c ; d, d e / b c ; q, q),
$$

to $\psi={ }_{3} \phi_{2}(a, d / b, d / c ; q d, d e / b c ; q, q)$ yields the following relations:

$$
\begin{aligned}
& \phi=\frac{(d e / b c ; q)_{n}}{(e ; q)_{n}}(b c / d)^{n} \psi\left(d^{-}\right), \\
& \phi_{+}=\frac{(d e / b c ; q)_{n-1}}{(q e ; q)_{n-1}}(q b c / d)^{n-1} \psi\left(a^{+}\right), \\
& \phi_{+}\left(a^{-}\right)=\frac{(d e / b c ; q)_{n}}{(q e ; q)_{n}}(q b c / d)^{n} \psi .
\end{aligned}
$$

Thus, from (2.7), changing $\phi$ into $\psi$, and using (2.10) we get

$$
\begin{aligned}
& d(1-a)\left\{\frac{(q e ; q)_{n-1}}{(d e / b c ; q)_{n-1}}(d / q b c)^{n-1} \phi_{+}-\frac{(q e ; q)_{n}}{(d e / b c ; q)_{n}}(d / q b c)^{n} \phi_{+}\left(a^{-}\right)\right\} \\
& -a(1-d)\left\{\frac{(e ; q)_{n}}{(d e / b c ; q)_{n}}(d / b c)^{n} \phi-\frac{(q e ; q)_{n}}{(d e / b c ; q)_{n}}(d / q b c)^{n} \phi_{+}\left(a^{-}\right)\right\}=0 .
\end{aligned}
$$

After some simplification this yields

$$
a(1-d)(1-e) \phi-(a-e)(a-d) \phi_{+}\left(a^{-}\right)-(1-a)(q a b c-d e) \phi_{+}=0 .
$$

From the symmetries of the ${ }_{3} \phi_{2}$, we have

$$
b(1-d)(1-e) \phi-(b-e)(b-d) \phi_{+}\left(b^{-}\right)-(1-b)(q a b c-d e) \phi_{+}=0 .
$$

Using (2.11), (2.12), and (2.1), we obtain the following contiguous relations:

$$
\begin{gathered}
a b(a-b)(1-c) \phi+b(a-e)(a-d)\left\{\phi\left(a^{-}\right)-\phi\right\} \\
-a(b-e)(b-d)\left\{\phi\left(b^{-}\right)-\phi\right\}=0, \\
q a^{2}(1-b)(1-c) \phi+q(a-e)(a-d)\left\{\phi\left(a^{-}\right)-\phi\right\} \\
-(1-a)(q a b c-d e)\left\{\phi\left(a^{+}\right)-\phi\right\}=0, \\
q b^{2}(1-b)(1-c) \phi+q(b-e)(b-d)\left\{\phi\left(b^{-}\right)-\phi\right\} \\
-(1-b)(q a b c-d e)\left\{\phi\left(b^{+}\right)-\phi\right\}=0 .
\end{gathered}
$$

Now, replacing $b$ by $b / q$ in (2.6) and $b$ by $b q$ in (2.13) we get

$$
\begin{aligned}
& (b-a q)\left\{\phi\left(b^{-}\right)-\phi\right\}+b(1-a) \phi-b(1-a) \phi\left(a^{+}, b^{-}\right)=0, \\
& (a-q b)(e d-q a b c)\left\{\phi\left(b^{+}\right)-\phi\right\}+b q(a-e)(a-d) \phi\left(a^{-}, b^{+}\right) \\
& \quad+\{(a-q b)(e d-q a b c)-a(q b-e)(q b-d)\} \phi=0 .
\end{aligned}
$$


Hence, combining (2.16) and (2.17) yields the three term contiguous relation

$$
\begin{aligned}
(a-e)(a-d)(1-b)(b-q a) \phi\left(a^{-}, b^{+}\right) \\
\quad-(1-a)(b-e)(b-d)(a-q b) \phi\left(a^{+}, b^{-}\right) \\
\quad+\left\{d(1-a)\left[a(e-b)+q b^{2}\right]+[2] a b(e-c)(a-b)-[2] b(e d+q b a)\right. \\
\quad+q b a^{2}([2]-a-d)+q(b-a)(q b a c+e(b+a)) \\
\left.\quad+b^{3}(q a-c)+q a^{2}(a c-d)+e d\left(q a+b^{2}\right)\right\} \phi=0 .
\end{aligned}
$$

\section{The reduction formula}

In this section, we state and prove the reduction formula for the $q$-integral $I_{n, q}$ stated in the introduction. We start with the following result.

Proposition 3.1 The q-integral $I_{n, q}$ can be represented in terms of basic hypergeometric series ${ }_{3} \phi_{2}$, that is,

$$
I_{n, q}=S_{n 3} \phi_{2}\left(q^{-n}, q^{-\beta-\delta-\alpha-\gamma+n-1}, q^{-\beta} ; q^{-\beta-\delta}, q^{-\alpha-\beta} ; q, q\right)
$$

where

$$
S_{n}=q^{\frac{n}{2}(2 \beta+5-n)} \frac{\Gamma_{q}(\beta+\delta+1) \Gamma_{q}(\alpha+\gamma+1)}{\Gamma_{q}(\alpha+\gamma+\beta+\delta-n+2)} \frac{\left(q^{-\gamma} ; q\right)_{n}\left(q^{-\alpha-\beta} ; q\right)_{n}}{\left(q^{-\alpha-\gamma} ; q\right)_{n}\left(q^{1-n+\gamma} ; q\right)_{n}} .
$$

Proof Calculating $D_{q^{-1}}^{n}\left[x^{\gamma}\left(q^{\beta+1} x ; q\right)_{\delta}\right]$ by using a $q^{-1}$-type Leibiniz rule (see [6], p.27) gives

$$
I_{n, q}=\int_{0}^{1} x^{\alpha}(q x ; q)_{\beta} \sum_{k=0}^{n}\left[\begin{array}{l}
n \\
k
\end{array}\right]_{q^{-1}}\left(D_{q^{-1}}^{n-k} f\right)\left(q^{-k} x\right) D_{q^{-1}}^{k} g(x) d_{q} x
$$

where

$$
f(x)=x^{\gamma} \quad \text { and } \quad g(x)=\left(q^{\beta+1} x, q\right)_{\delta}
$$

Note that

$$
\begin{aligned}
& \left\{D_{q^{-1}}^{n-k}(\cdot)^{\gamma}\right\}\left(q^{-k} x\right)=\frac{(-q)^{n-k}\left(q^{-\gamma} ; q\right)_{n-k}}{(1-q)^{n-k}} q^{-k^{2}+n k-\gamma k} x^{\gamma-n+k}, \\
& D_{q^{-1}}^{k}\left(q^{\beta+1} x, q\right)_{\delta}=q^{(\beta+\delta+1) k} \frac{q^{-\frac{1}{2} k(k-1)}}{(1-q)^{k}}\left(q^{\beta+1} x, q\right)_{\delta-k}\left(q^{-\delta}, q\right)_{k} .
\end{aligned}
$$

This implies

$$
\begin{aligned}
I_{n, q}= & \sum_{k=0}^{n}\left[\begin{array}{l}
n \\
k
\end{array}\right]_{q^{-1}} \frac{(-q)^{n-k}\left(q^{-\gamma} ; q\right)_{n-k}}{(1-q)^{n}}\left(q^{-\delta} ; q\right)_{k} q^{-\frac{1}{2} k(k-1)} \\
& \times q^{(\beta+\delta+1) k-(k-n+\gamma) k} \int_{0}^{1} x^{\alpha+\gamma-n+k}(q x ; q)_{\beta}\left(q^{\beta+1} x ; q\right)_{\delta-k} d_{q} x .
\end{aligned}
$$


Denoting the $q$-integral in the left-hand-side of (3.1) by $J_{n}$, we obtain

$$
\begin{aligned}
J_{n} & =\int_{0}^{1} x^{\alpha+\gamma-n+k}(q x ; q)_{\beta+\delta-k} d_{q} x \\
& =B_{q}(\alpha+\gamma-n+k+1, \beta+\delta-k+1) \\
& =\frac{\Gamma_{q}(\alpha+\gamma-n+k+1) \Gamma_{q}(\beta+\delta-k+1)}{\Gamma_{q}(\alpha+\gamma+\beta+\delta-n+2)} .
\end{aligned}
$$

Using [6], Eq. (I.8) and Eq. (I.35), we get

$$
J_{n}=\frac{\Gamma_{q}(\alpha+\gamma+1) \Gamma_{q}(\beta+\delta+1)}{\Gamma_{q}(\alpha+\gamma+\beta+\delta-n+2)} \frac{(1-q)^{n} q^{k^{2}-n k+\frac{1}{2} n(n+1)} q^{-(\beta+\delta) k-(n-k)(\alpha+\gamma)}}{(-1)^{n}\left(q^{-\beta-\delta} ; q\right)_{k}\left(q^{-\alpha-\gamma} ; q\right)_{n-k}}
$$

Using [6], Eq. (I.11), we get

$$
\frac{\left(q^{-\gamma} ; q\right)_{n-k}}{\left(q^{-\alpha-\gamma} ; q\right)_{n-k}}=\frac{\left(q^{-\gamma} ; q\right)_{n}}{\left(q^{-\alpha-\gamma} ; q\right)_{n}} \frac{\left(q^{1-n+\alpha+\gamma} ; q\right)_{k}}{\left(q^{1-n+\gamma} ; q\right)_{k}} q^{-\alpha k}
$$

Substituting (3.2) into (3.1), using (3.3), yields

$$
\begin{aligned}
I_{n, q}= & \frac{\Gamma_{q}(\alpha+\gamma+1) \Gamma_{q}(\beta+\delta+1)}{\Gamma_{q}(\alpha+\gamma+\beta+\delta-n+2)} q^{n(1-\gamma-\alpha)+\frac{1}{2} n(n+1)} \frac{\left(q^{-\gamma} ; q\right)_{n}}{\left(q^{-\alpha-\gamma} ; q\right)_{n}} \\
& \times \sum_{k=0}^{n}\left[\begin{array}{l}
n \\
k
\end{array}\right]_{q^{-1}}(-1)^{k} q^{-\frac{1}{2} k(k-1)} \frac{\left(q^{1-n+\alpha+\gamma} ; q\right)_{k}\left(q^{-\delta} ; q\right)_{k}}{\left(q^{1-n+\gamma} ; q\right)_{k}\left(q^{-\delta-\beta} ; q\right)_{k}} .
\end{aligned}
$$

Now using [6], Eq. (I.42) and Eq. (I.47), we get

$$
\begin{aligned}
I_{n, q} & =K_{n} \sum_{k=0}^{n} q^{k} \frac{\left(q^{-n}, q^{-\delta}, q^{1-n+\alpha+\gamma} ; q\right)_{k}}{\left(q^{-\beta-\delta}, q, q^{1-n+\gamma} ; q\right)_{k}} \\
& =K_{n 3} \phi_{2}\left(q^{-n}, q^{1-n+\alpha+\gamma}, q^{-\delta} ; q^{-\beta-\delta}, q^{1-n+\gamma} ; q, q\right),
\end{aligned}
$$

where

$$
K_{n}=\frac{\Gamma_{q}(\beta+\delta+1) \Gamma_{q}(\alpha+\gamma+1)}{\Gamma_{q}(\alpha+\gamma+\beta+\delta-n+2)} q^{n(1-\gamma-\alpha)+\frac{1}{2} n(n+1)} \frac{\left(q^{-\gamma} ; q\right)_{n}}{\left(q^{-\alpha-\gamma} ; q\right)_{n}} .
$$

Using the transformation (2.9) yields the required result and completes the proof.

Corollary 3.2 If $\gamma=0$ then $I_{n, q}$ vanishes for all values of $n$ where $n-\beta-\delta$ and $\beta$ are nonnegative integers.

Proof Since

$$
{ }_{3} \phi_{2}\left(a, b_{1} q^{m_{1}}, b_{2} q^{m_{2}} ; b_{1}, b_{2} ; q, a^{-1} q^{-\left(m_{1}+m_{2}\right)}\right)=0,
$$

where $m_{1}, m_{2}$ are arbitrary nonnegative integers (see [6]), the proof follows directly from Proposition 3.1 and (3.5). 
Watson remarked $I_{n}$ vanishes for odd values of $n$ in two special cases, (i) $\alpha=\gamma$ and $\beta=\delta$ and (ii) $\alpha=\beta$ and $\gamma=\delta$.

Now, we can derive the reduction formula for $I_{n, q}$.

Theorem 3.3 The reduction formula satisfies a three term recurrence relations of $I_{n, q}$. More precisely, the following holds:

If

$$
W_{n}={ }_{3} \phi_{2}\left(q^{-n}, q^{-\beta-\delta-\alpha-\gamma+n-1}, q^{-\beta} ; q^{-\beta-\delta}, q^{-\alpha-\beta} ; q, q\right),
$$

then

$$
L_{n} W_{n+1}-Q_{n} W_{n-1}+M_{n} W_{n}=0
$$

where

$$
\begin{aligned}
L_{n}= & \left(q^{-n}-q^{\theta_{1}}\right)\left(q^{-n}-q^{\theta_{2}}\right)\left(1-q^{\theta_{3}+n}\right)\left(q^{\theta_{3}+n}-q^{1-n}\right), \\
Q_{n}= & \left(1-q^{-n}\right)\left(q^{\theta_{3}+n}-q^{\theta_{1}}\right)\left(q^{\theta_{3}+n}-q^{\theta_{2}}\right)\left(q^{-n}-q^{\theta_{3}+n+1}\right), \\
M_{n}= & q^{\theta_{2}}\left(1-q^{-n}\right)\left[q^{-n}\left(q^{\theta_{1}}-q^{\theta_{3}+n}\right)+q^{2\left(\theta_{3}+n\right)+1}\right]+q^{3\left(\theta_{3}+n\right)}\left(q^{1-n}-q^{-\beta}\right) \\
& -[2] q^{\theta_{3}+n}\left(q^{\theta_{1}+\theta_{2}}+q^{1+\theta_{3}}\right)+q^{1+\theta_{3}-n}\left([2]-q^{-n}-q^{\theta_{2}}\right)+q^{1-2 n}\left(q^{-n-\beta}-q^{\theta_{2}}\right) \\
& +q\left(q^{\theta_{3}+n}-q^{-n}\right)\left(q^{1+\theta_{3}-\beta}+q^{\theta_{1}}\left(q^{\theta_{3}+n}+q^{-n}\right)\right)+q^{\theta_{1}+\theta_{2}}\left(q^{1-n}+q^{2\left(\theta_{3}+n\right)}\right) \\
& +[2] q^{\theta_{3}}\left(q^{\theta_{1}}-q^{-\beta}\right)\left(q^{-n}-q^{\theta_{3}+n}\right),
\end{aligned}
$$

and $\theta_{1}=-\alpha-\beta, \theta_{2}=-\delta-\beta, \theta_{3}=-\alpha-\beta-\delta-\gamma-1$.

Proof This result follows by applying Proposition 3.1 and using equation (2.18) with

$$
b=q^{-\beta-\delta-\alpha-\gamma+n-1}, \quad c=q^{-\beta}, \quad d=q^{-\beta-\delta} \quad \text { and } \quad e=q^{-\alpha-\beta} .
$$

Recall that the little $q$-Jacobi polynomials, see [16], are defined by

$$
P_{n}(x ; a, b ; q)={ }_{2} \phi_{1}\left(q^{-n}, a b q^{n+1} ; a q ; q, q x\right),
$$

and the formula

$$
P_{n}(x ; c, d ; q)=\sum_{k=0}^{n} a_{k, n} P_{k}(x ; a, b ; q)
$$

holds with

$$
\begin{aligned}
& a_{k, n}=C_{k 3} \phi_{2}\left(q^{k-n}, c d q^{n+k+1}, a q^{k+1} ; c q^{k+1}, a b q^{2 k+2} ; q, q\right), \\
& C_{k}=(-1)^{k} q \frac{\left(q^{-n}, a q, c d q^{n+1} ; q\right)_{k}}{\left(q, c q, a b q^{k+1} ; q\right)_{k}} .
\end{aligned}
$$


Remark 3.4 In (3.7), if we take $a=q^{-\beta-1}, b=q^{-\alpha-1}, c=q^{-\beta-\delta-1}$ and $d=q^{-\alpha-\gamma-1}$, we get $a_{0, n}=\frac{1}{S_{n}} I_{n, q}$. Thus, the little $q$-Jacobi polynomials and the $q$-integrals $I_{n, q}$ are related in the following way:

$$
P_{n}(x ; c, d ; q)=\frac{1}{S_{n}} I_{n, q}+\sum_{k=1}^{n} a_{k, n} P_{k}(x ; a, b ; q) .
$$

\section{Competing interests}

The authors declare that they have no competing interests.

\section{Authors' contributions}

The authors have made equal and significant contributions in writing this paper. They read and approved the final manuscript.

\section{Author details}

${ }^{1}$ Present address: Department of Mathematics, Faculty of Science, King Saud University, Riyadh, KSA. ${ }^{2}$ Permanent address: Department of Mathematics, Faculty of Science, Cairo University, Giza, Egypt. ${ }^{3}$ Department of Natural and Engineering Science, Faculty of Applied Studies and Community Service, King Saud University, Riyadh, KSA.

\section{Acknowledgements}

The authors would like to thank Prof. Mourad Ismail for pointing out the relation between $I_{n, q}$ and the little $q$-Jacobi polynomials. They are very grateful to the reviewers and editors for their suggestions and comments which improved final version of this paper. This research is supported by the DSFP program of the King Saud University in Riyadh through grant DSFP/MATH 01.

Received: 25 October 2015 Accepted: 13 March 2016 Published online: 22 March 2016

\section{References}

1. Watson, GN: A reduction formula. Proc. Glasgow Math. Assoc. 2, 57-61 (1954)

2. Bailey, WN: Contiguous hypergeometric functions of the type ${ }_{3} F_{2}(1)$. Proc. Glasgow Math. Assoc. 2, $62-65$ (1954)

3. Annaby, MH, Mansour, ZS: q-Fractional Calculus and Equations, vol. 2056. Springer, Berlin (2012)

4. Rainville, ED: Special Functions. The MacMillan Company, New York (1960)

5. Andrews, GE, Askey, R, Roy, R: Special Functions. Cambridge University Press, Cambridge (1999)

6. Gasper, G, Rahman, M: Basic Hypergeometric Series. Cambridge University Press, Cambridge (2004)

7. Jackson, FH: The basic gamma function and elliptic functions. Proc. R. Soc. A 76, 127-144 (1905)

8. Jackson, FH: On q-definite integrals. Q. J. Pure and Appl. Math. 41, 193-203 (1910)

9. Askey, R: The q-gamma and $q$-beta functions. Appl. Anal. 8(2), 125-141 (1979)

10. Wilson, JA: Hypergeometric series, recurrence relations and some orthogonal functions. Ph.D. diss., University of Wisconsin, Madison (1978)

11. Gupta, DP, Ismail, ME, Masson, DR: Contiguous relations, basic hypergeometric functions, and orthogonal polynomials. II. Associated big q-Jacobi polynomials. J. Math. Anal. Appl. 2, 477-497 (1992)

12. Gautschi, W: Computational aspects of three-term recurrence relations. SIAM Rev. 9, 24-82 (1967)

13. Rakha, MA, Ibrahim, AK: On the contiguous relations of hypergeometric series. J. Comput. Appl. Math. 192(2), 396-410 (2006)

14. Ismail, ME, Masson, DR: Generalized orthogonal and continued fractions. J. Approx. Theory 83, 1-40 (1995)

15. Vidūnas, R: Contiguous relations of hypergeometric series. J. Math. Anal. Appl. 135, 507-519 (2003)

16. Andrews, GE, Askey, R: Enumeration of partitions: the role of Eulerian series and q-orthogonal polynomials. In: Aigner, M (ed.) Higher Combinatorics, pp. 3-26. Reidel, Boston (1977)

\section{Submit your manuscript to a SpringerOpen ${ }^{\circ}$ journal and benefit from:}

- Convenient online submission

Rigorous peer review

- Immediate publication on acceptance

- Open access: articles freely available online

- High visibility within the field

- Retaining the copyright to your article 